\title{
Unidades físicas, de José Muñoz del Casti- llo, primer libro de análisis dimensional en la historia
}

\author{
Muñoz del Castillo’s physical units, first \\ book on dimensional analysis in history.
}

\author{
ANDRÉS RIVADULLA ${ }^{1}$ \\ Universidad Complutense
}

Recibido: 20/11/2015 Aceptado: 02/02/2015

\section{RESUMEN}

«Ningún volumen se ha consagrado al análisis dimensional hasta 1922 -cien años después de la aparición del Traité de Fourier-cuando P. W. Bridgman publicó el curso que había impartido en la Universidad de Yale.» Así se expresa Monod-Herzen (1976, p. 25). Entre ambos libros, Fourier 1822 y Bridgman 1922, fue Maxwell quien abonó el terreno para la consolidación de esta disciplina, y su semilla no cayó en terreno baldío. En España, sin ir más lejos, la labor de Maxwell arraigó desde bien pronto. Hasta tal punto que la historia desmiente al epistemólogo francés. Pues sí que hay un libro sobre análisis dimensional entre Fourier y Bridgman. Se trata de Unidades Físicas del físico español José Muñoz del Castillo, que lo publica en 1890, o sea treinta y dos años antes de la publicación del libro de Bridgman. Así pues Unidades Físicas se convierte en el primer libro dedicado al análisis dimensional. Naturalmente no voy a cuestionar

1 Grupo de Investigación Complutense 930174 y Proyecto de Investigación FFI201452224-P del Ministerio de Economía y Competitividad del Reino de España.

Agradezco a un evaluador anónimo sus sugerencias sobre una versión anterior de este artículo, así como a Pablo Soler Ferrán su atenta lectura y acertados comentarios. 
que sea el Dimensional Analysis de Bridgman el primer tratado completo de esta disciplina. Pero no es el primer libro en el que se recoge el conocimiento de la teoría de las dimensiones misma, como podía ser conocida en el siglo XIX, veinticuatro años antes de la propuesta de Buckingham 1914 de su famoso Teorema $\pi$. El objeto de este artículo es remarcar la aceptación y desarrollo del análisis dimensional en la España del XIX, una circunstancia hasta ahora no suficientemente apreciada por la historiografía especializada.

PALABRAS CLAVE: ANÁLISIS DIMENSIONAL, MÉTODO DE DIMENSIONES, FOURIER, MAXWELL, GARAGARZA, MUÑOZ DEL CASTILLO

\begin{abstract}
«No book has been devoted to the dimensional analysis until 1922 -a hundred years after the appearance of Fourier's Traité - as P.W. Bridgman published the course he taught at Yale University.» This is what Monod-Herzen (1976, p. 25) affirms. Between both books, Fourier's 1822 and Bridgman's 1922, James Clerk Maxwell paved the way for the consolidation of this discipline, and his seed did not fall on barren ground. In Spain, without going any further, the work of Maxwell root from soon. So much history belies the French epistemologist. Yes, there is a book on dimensional analysis between Fourier and Bridgman. It is Physical Units written by the Spanish physicist José Muñoz del Castillo, who published it in 1890, that is thirty-two years before the publication of the book by Bridgman. Physical Units thus becomes the first treatise devoted to dimensional analysis. Naturally I will not question that Bridgman's Dimensional Analysis has been the first complete treatise on this discipline. But it is not the first book in which the theory of dimensions is presented, as it could be known in the nineteenth century, twenty four years before the proposal by Buckingham of his famous theorem $\pi$. The purpose of this article is to highlight the acceptance and development of dimensional analysis in Spain in the 19th century, a circumstance so far not sufficiently appreciated by the specialized historiography.

KEYWORDS: DIMENSIONAL ANALYSIS, METHOD OF DIMENSIONS, FOURIER, MAXWELL, GARAGARZA, MUÑOZ DEL CASTILLO.
\end{abstract}

\title{
I. ANTECEDENTES HISTÓRICOS
}

Algunos historiadores atribuyen el origen del análisis dimensional a Osborne Reynolds (1842-1912). A éste se debe el denominado 'número de Reynolds', que presentó por vez primera en 1883, y que se aplica en dinámica de fluidos. El número de Reynolds es el número adimensional $R=\frac{v d}{\eta} \rho$ donde $\eta$ es la viscosidad, $\rho$ es la densidad, $v$ la velocidad -todas del fluido- y $d$ es el diámetro del tubo. Como experimentalmente se concluye que, para $R<2.400$, el régimen de circulación del fluido por el tubo es laminar, mientras que para $R>2.400$ es turbulento, la fórmula del número de Reynolds permite calcular la velocidad crítica que marca la frontera del régimen de circulación de un fluido a través de un tubo. 
También se identifica al análisis dimensional con el Principle of Similitude de Richard C. Tolman², 1914, en relación al cual Lord Rayleigh (1915, p. 66) confiesa

Me ha impresionado la escasa atención que los físicos primeros han dedicado al gran principio de semejanza. No es infrecuente que, sobre la base de experimentos elaborados, se anticipen como novedades resultados en forma de 'leyes', que podrían haber sido predichos a priori tras unos pocos minutos de consideración.

Éste es un principio de gran importancia, a pesar de su descuido tanto por los matemáticos como por los ingenieros, sostiene Rayleigh, quien en su ejemplificación del problema de Boussinesq del «paso constante de calor de un buen conductor sumergido en una corriente de fluido en movimiento (a una distancia del sólido) con velocidad v». Rayleigh $(1915$, p. 67) recurre a las dimensiones de diferentes magnitudes: calor total que pasa por unidad de tiempo, dimensión lineal del sólido, diferencia de temperatura, velocidad de la corriente, capacidad calorífica del fluido por unidad de volumen y conductividad. Se puede incluir también la viscosidad cinemática cuyas dimensiones serían (Longitud) ${ }^{2}\left(\right.$ Tiempo) ${ }^{-1}$. Pues bien, como reconoce Buckingham (1915. Mis cursivas):

En su artículo (NATURE, 18 de marzo de 1915, p. 66) Lord Rayleigh deduce, por el método de dimensiones, una ecuación para la tasa de transferencia de calor entre un cuerpo sólido y una corriente de fluido en la que está inmerso. Comentando esta ecuación, M. Riabouchinsky (NATURE, 29 de julio, p 591) señala que el calor, la temperatura, la longitud y el tiempo son tratados en la deducción como unidades independientes; y que si suponemos que sólo tres de estas unidades son 'realmente independientes' obtenemos un resultado diferente y menos definido.

El análisis dimensional, o método dimensional, precede al principio de semejanza. Esto es obvio por el uso que hacen de él Buckingham y Rayleigh. Y por supuesto, es muy anterior al descubrimiento del número de Reynolds. Por otra parte, la terminología que se usa para mencionarlo es la de método dimensional. Análisis dimensional es una forma tardía de referirse a él. Ésta no la usan ni Maxwell, quien emplea la de theory of dimensions, ni Buckingham, quien hace uso frecuente de dimensional reasoning, pero también de method of dimensions, ni tampoco Lord Rayleigh. Alfred W. Porter (1933) también usa la expresión method of dimensions ${ }^{3}$. E igualmente la emplea Ricardo San Juan, ya

2 Un tratamiento extenso del mismo lo realiza González Redondo (2000, Cap. 8)

3 De hecho, su libro lleva precisamente por título The Method of Dimensions y constituye «el primer tratado (ipor pequeño que sea!) sobre este asunto publicado en Gran Bretaña» (Porter 1933, Preface) 
consolidada la de análisis dimensional, quien sigue usando la expresión método dimensional. En efecto, el siguiente texto de San Juan (1945, p. 180) evidencia que la terminología es independiente del estadio avanzado de la disciplina: «El teorema de $\pi$ suministra un procedimiento, denominado método dimensional, para obtener expresiones abstractas de las leyes que rigen los fenómenos físicos, o sea de los teoremas a que se refiere su enunciado.»

El análisis dimensional constituye en sus orígenes un cálculo para el establecimiento de las dimensiones de las magnitudes físicas. Cualquier magnitud derivada, por ejemplo la aceleración, se puede expresar en términos de unidades fundamentales, como espacio $[L]$ y tiempo $[T]: A=\left[L T^{-2}\right]$. A esta expresión se la denomina fórmulalecuación dimensional, mientras que los exponentes de la misma constituyen las dimensiones de la magnitud en cuestión en relación a la base de unidades fundamentales (Palacios 1964, p. 56). Es una regla de obligado cumplimiento en la metodología de la física que las ecuaciones que expresan una ley sean dimensionalmente homogéneas, o sea, que los dos miembros de la ecuación tengan la misma fórmula dimensional. En reiteradas ocasiones Ricardo San Juan se refiere a la Regla de la Homogeneidad. Así, en (1945, p. 140) afirma «Es, pues, condición necesaria, pero no suficiente, para la veracidad de una igualdad entre medidas, que sus dos miembros tengan la misma dimensión. Esta propiedad se llama homogeneidad de la ecuación y debe comprobarse, como es sabido, para desechar ésta si no se cumple.» Y en (op. cit., ibid.) San Juan reitera que «la homogeneidad de una fórmula entre medidas, esto es, la igualdad de las dimensiones de los dos miembros... es condición necesaria pero no suficiente para su veracidad.»

También insiste Ricardo San Juan (1945, p. 123, nota 6) en la diferencia entre la fórmula dimensional de una magnitud y su dimensión:

La fórmula dimensional es una expresión monomia que depende evidentemente de la base elegida, mientras que la dimensión es un ente abstracto... una misma dimensión puede tener expresiones diversas, que son fórmulas dimensionales distintas, según la base que se adopte,... Quizá extrañe esta distinción a los físicos acostumbrados a considerar como dimensión la fórmula dimensional.

Y en (1945, p. 131) añade además que «las dimensiones no dan la esencia de las magnitudes, o sea su naturaleza, compuesta de las propiedades geométricas, físicas, químicas, etc.,... podemos agregar que ni siquiera dan sus propiedades algebraicas.»

Sobre el Postulado General de Homogeneidad González Redondo (2000, Cap. 27) ofrece una interesante retrospectiva histórica, pues se remonta a François Viète, 1591.

De forma generalmente aceptada se debe a Fourier 1822, $\S 160,161$ la introducción del concepto de dimensión de una magnitud y del de homogenei- 
dad dimensional de una ecuación, y es él quien da, por vez primera una tabla de dimensiones. Así lo reconoce James Clerk Marwell en una carta a William Thomson el 14 de April de 1870: «La primera tabla de dimensiones que conozco se encuentra en Fourier, Théorie de Chaleur p. 157, y él hace uso frecuente de la misma. Las dimensiones son de longitud, tiempo y temperatura ${ }^{4}$.»

Seis años más tarde, en una carta a los síndicos de la Cambridge University Press $^{5}$, Maxwell afirma:

El tratado de Fourier es uno de los pocos libros científicos que nunca pueden quedar anticuados por el progreso de la ciencia. No es sólo el primero y más grande libro sobre la física de la conducción de calor, sino que en cada capítulo nuevas perspectivas se abren a vastos campos de la especulación matemática.

Y en (1871, p. 258) Maxwell reitera de nuevo sus elogios a la 'Theory of Heat' de Fourier, de la que esta obra de Maxwell de 1871 toma su título. Respecto a la preeminencia de Fourier en el análisis dimensional, Maxwell (1877, p. 518) (Mis cursivas) reconoce que «La teoría de las dimensiones de las magnitudes físicas (fue) formulada primeramente por Fourier, Théorie de Chaleur, sec. 160.»

De hecho, sería posible remontarse a 1761, al texto de Daviet de Foncenex «Sur les principes fondamentaux de la mécanique» ${ }^{6}$, para encontrar la primera referencia al término de dimensión. González Redondo $(2000, \S 31.2)$ presenta a Foncenex como un precedente, cuyo trabajo el propio Fourier conocía. Martins (2008) analiza detalladamente el artículo de Foncenex, quien en su trabajo no explica el concepto de dimensión que estaba empleando. Martins (2008, pp. 395-396) emprende en su artículo la tarea de ofrecer una reconstrucción conceptual del concepto de dimensión de Foncenex, y sus posibles repercusiones, en particular en Poisson. Pero reconoce que fue Fourier quien «desarrolló, más adelante, un análisis más adecuado del concepto de dimensión física.» (Martins 2008, p. 401). Es la misma conclusión a la que llega Monod-Herzen (1976, p. 24): «fue (Fourier) quien hizo la primera exposición teórica del análisis dimensional y es a partir de esta exposición que se desarrolló esta teoría físico-matemática.»

Afirma González de Posada (2002, pp. 403 y 418, nota 11) que Palacios había tomado conocimiento del Análisis Dimensional como teoría científica a partir del libro de W. S. Hill Teoría General de las Magnitudes Físicas, de

4 The Scientific Letters and Papers of James Clerk Maxwell, Vol. II. También Palacios (1964, p. 10) reproduce la Tabla de Fourier.

5 The Scientific Letters and Papers of James Clerk Maxwell, Vol. III.

6 «Mélanges de philosophie et de mathématique de la Société Royal de Turin pour les années 1760-1761», pp. 299-322. Citado por Monod-Herzen (1976, p. 24). 
1941. Esta obra está también muy presente en Ricardo San Juan (1945). Julio Palacios (1964, p. 9) comienza la Introducción de su libro con las siguientes palabras: «Se debe al barón Jean Batiste Fourier el haber aplicado a las magnitudes físicas el concepto geométrico de dimensión y, por ello, merece ser considerado como el precursor del Análisis dimensional.» Unas líneas más abajo, Palacios continúa: «Las ideas de Fourier fueron aplicadas con gran éxito a fines del pasado siglo por Reynolds, Lodge, FitzGerald, Rücker, Jeans, y, muy especialmente, por lord Rayleigh ${ }^{7}$.... Lord Rayleigh... merece ser considerado, después de Fourier, como el fundador del Análisis dimensional.»

En esta relación histórica brilla por su ausencia el nombre de James Clerk Maxwell. Sin embargo de éste dice Monod-Herzen (1976, p. 24) que la idea de formar fórmulas dimensionales no procede de Fourier, sino que se debe a Maxwell. Cosa que el propio Palacios $(1964$, p. 12) implícitamente reconoce cuando asevera que

Maxwell atribuyó a cada magnitud, $Y$, una fórmula dimensional: $[\mathrm{Y}]=\left[\mathrm{M}_{1}\right]^{\alpha 1}$ $\left[\mathrm{M}_{2}\right]^{\alpha 2} \ldots\left[\mathrm{M}_{\mathrm{m}}\right]^{\alpha \mathrm{m}}$ en la que $M_{l}, M_{2}, \ldots$ son los símbolos de las magnitudes que

forman la base, y $\alpha_{1} \ldots \alpha_{m}$ son los exponentes dimensionales de $Y$.

Si bien -una ausencia incomprensible que podría explicar el salto que da entre Fourier y Rayleigh - en la bibliografía de su libro cualquier referencia a Maxwell brilla por su ausencia. Teniendo en cuenta el papel de Rayleigh en la divulgación de la obra de Maxwell esto sorprende.

Pues el caso es que Maxwell introdujo ya la notación dimensional en el artículo escrito en colaboración con Fleeming Jenkin titulado «On the elementary relations of electrical quantities», 1863. A partir de entonces el uso de dimensiones por parte de Maxwell es amplísimo. Así, en varias cartas a William Thomson, hace uso de él. Por ejemplo, dos años después, en una carta del 17 de Abril de 1865 a Lord Kelvin ${ }^{8}$ sobre la fricción de los gases, Maxwell deduce las dimensiones del coeficiente de fricción .

$$
\mu=\frac{\text { Force } / \text { area }}{\text { velocity } / \text { dist }}=\frac{M L / L^{2} T^{2}}{L / T L}=\frac{M}{L T}
$$

En otra carta de 27 de Febrero de 1866 reitera también las mismas dimensiones para este coeficiente. Pero es en sus libros Theory of Heat, 1871, y A Treatise on Electricity and Magnetism, 1873, donde aplica ampliamente el

7 Las obras citadas de estos autores abarcan el período de 1883 a 1905.

8 Maxwell, Vol. II, p. 218. 
cálculo de dimensiones. Por ejemplo en 1871, Cap. XVIII, en la sección «On the dimensions of $k$, the specific termal conductivity», Maxwell da la fórmula dimensional de $k$ en la base: unidad de longitud, tiempo, calor y temperatura. En el capítulo XX, Sección «Capillarity», p. 283, da las dimensiones de la energía superficial de una pompa de jabón: $\mathrm{M} / \mathrm{T}^{2}$. Y en p. 305 indica que las dimensiones del coeficiente de viscosidad de una sustancia «son $\left[M L^{-1} T^{-1}\right]$, donde $M, L$, y $T$ son las unidades de masa, longitud y tiempo.»

«Toda magnitud electromagnética puede ser definida por referencia a las unidades fundamentales de Longitud, Masa y Tiempo.» Así comienza Maxwell el Capítulo X, «Dimensions of Electric Units» de su Treatise on Electricity and Magnetism de 1873. En la sección 628 presenta las dimensiones (fórmulas dimensionales) de doce magnitudes, de las que asevera (\$ 624): «Todas las relaciones dadas anteriormente son verdaderas independientemente del sistema de unidades que adoptemos.»

Y en $\$ 626$ presenta la Table of Dimensions de diez magnitudes en los sistemas electrostático y electromagnético. Pero ya en la sección 374 había mostrado las dimensiones de la unidad polo magnético de las que afirma que son las mismas que las de la unidad electrostática de electricidad. Finalmente, en (1877, p. 518) Maxwell reitera que «Conociendo las dimensiones de cualquier magnitud somos capaces de inmediato de deducir su valor numérico expresado en términos de un sistema de unidades a partir de su valor numérico dado en términos de otro sistema.»

Además, las dimensiones de las magnitudes pueden expresarse también unas en términos de otras. Así, partiendo de que las dimensiones de la unidad de densidad $[D]$ son $\left[M L^{-3}\right]$ y las de la unidad de presión $[P]$ son $\left[M L^{-1} T^{-2}\right]$, entonces las dimensiones de $[L]$ son $\left[\mathrm{P}^{1 / 2} D^{-1 / 2} T\right]$ y las de $[M]$ son $\left[\mathrm{P}^{3 / 2} D^{-1 / 2} T^{3}\right]$, siendo $M, L$ y $T$ (masa, espacio y tiempo) independientes entre sí.

Pero la investigación histórica todavía depara otra sorpresa. La única referencia histórica relevante que hace Porter (1933, Cap. I-A. Chiefly Historical) se restringe a Fourier cuyos conceptos de dimensión y requisito de homogeneidad presenta. En el Prefacio afirma que «Nadie ha hecho más en Gran Bretaña para fomentar el desarrollo de la materia que el tercer Lord Rayleigh.»

Menciona Porter (1933, p. 63) además la discusión RiabouchinskyRayleigh, así como el artículo de Arthur Rücker de 1899, pero Maxwell, en relación al método dimensional, brilla también por su ausencia.

\section{FAUSTO GARAGARZA Y EL ANÁLISIS DIMENSIONAL EN LA ESPAÑA DEL SIGLO XIX}

En Rivadulla (2015) dedico el capítulo VII a exponer la teoría de la preducción, con la que se implementa la aplicación del razonamiento deductivo a la creatividad en física teórica. Como esta práctica metodológica considera 
fundamental que las combinaciones de resultados aceptados en física, que actúan como premisas del razonamiento deducido, respeten escrupulosamente el método dimensional, hago en Rivadulla (2015, p. 189 nota 1) una brevísima referencia a este procedimiento y a su historia, en la que destaca la figura de Fausto Garagarza, un físico español del siglo XIX.

Con ocasión del centenario de la Real Sociedad Española de Física (19032003) José Manuel Sánchez Ron escribe un breve artículo sobre la Física en España en el siglo XIX. En su contribución (2003, p. 7) Sánchez Ron cita el discurso de Gumersindo Vicuña, "Cultivo actual de las ciencias físicomatemáticas en España», de apertura del curso académico 1875-1876 en la Universidad Central donde Vicuña constataba «el gran atraso en que se halla el cultivo de la Física en España comparada con el de otras ciencias».

«¿Cuál era el tipo de físico que predominaba en la España del Ochocientos?» es la pregunta a la que Sánchez Ron (2003) se enfrenta. La relación de físicos que menciona no es amplia, y el nombre de Garagarza no aparece. En todo caso, en opinión de Sánchez Ron (1992 y 2003), la física española del XIX carece de originalidad y además era 'muy pobre'. Bueno, esta era la imagen que el testigo privilegiado Gumersindo Vicuña trasladaba, que le sirve a Sánchez Ron de hilo conductor de su presentación de 1992, sobre la que construye la de 2003. No obstante en ambas memorias se afirma que «En la última década del siglo XIX comenzaron a observarse signos esperanzadores relativos al desarrollo de la física en España.»

Bien, sin pretender cuestionar la autoridad de Vicuña y su conocimiento de la situación, quizás el análisis dimensional sí se encontraba a la altura. En efecto, aunque ha pasado inadvertido a los historiadores contemporáneos, Fausto Garagarza merece un lugar de honor en la historia de la física española del XIX. Fijo mi atención en él en particular por su exposición del análisis dimensional al nivel que se podría esperar a principios de los años noventa del siglo XIX.

Fausto Garagarza y Dujiols (1833-1905) fue catedrático desde 1867, y decano en la Facultad de Farmacia de la Universidad de Santiago de Compostela. En el interim accede al cargo de Gobernador/Presidente de la Diputación de la Coruña durante el reinado de don Amadeo I de Saboya. Se traslada en 1876 a la Universidad Central de Madrid, de cuya Facultad de Farmacia también fue decano en dos períodos. El más largo, de 1887 a 1900, año de su jubilación. En el año 1892 se crea la cátedra de Física en la Facultad de Farmacia y en este año publica su libro Instrumentos y aparatos de Física, que fue libro de texto. Lo fue, pues, durante trece años al menos, y según se dice en El museo de la farmacia hispana, de 1993, además fue «obra de referencia obligatoria por numerosos profesionales.» Parece pues, que debió de dejar huella, aunque el paso del tiempo pudo borrarla. 
Pero el contenido del libro de Garagarza no se corresponde en absoluto con su título. Está compuesto de diez capítulos: Cap. I. Valores numéricos de las cantidades y unidades físicas, p. 27. Cap. II Sin título, dividido en grupos: 1. Cantidades y unidades geométricas. 2. Cantidades y unidades mecánicas. 3. Unidades magnéticas. 4. Unidades y cantidades eléctricas. Cap. III. Inercia. Movimiento. Fuerza viva. Trabajo y energía. Conservación de la energía, p. 49. Cap. IV. Leyes experimentales del péndulo. Leyes de la caída de los cuerpos, p. 53. Cap. V. Peso. Densidad. Densidad relativa. Peso específico. Masa considerada como coeficiente de resistencia. Balanzas. Areómetros. Densímetros, p. 55. Cap. VI. Presión ejercida por la atmósfera. Barómetro de mercurio. Tubos de seguridad. Corrección de volumen en los gases. Trompas. Densidad de los vapores, p. 73. Cap. VII. Temperatura. Calórico de los cuerpos. Coeficiente de dilatación de los líquidos, p. 88. Cap. VIII. Acciones moleculares. Cohesión. Afinidad. Elasticidad. Vibraciones. Capilaridad, p. 108. Cap. IX. Electricidad, p. 129. Cap. X. Óptica. Luz, p. 195. Estos capítulos se desglosan en un total de ochenta secciones que desarrollan, como se ve por los títulos de los capítulos, los principios fundamentales o generales de la física. Se trata pues de un libro de física en toda regla, casi de física teórica, y no, como parecería desprenderse del título de la asignatura, de la que pretende ser el programa, de un libro sobre instrumentos y aparatos de aplicación a la farmacia. El libro concluye con 16 láminas en las que aparecen representados aparatos y esquemas de procesos físicos: 1. Balanza de precisión de brazos cortos, de Sartorius. 2. Balanza hidrostática de Mohr. 3. Barómetro de mercurio y máquina neumática de mercurio de Alvergniat. 4. Matraz productor de gas y aparato de Wolf. 5. Trompa de vacío de Christiansen y Lasne y dos aparatos más de Alvergniat, uno de ellos para mantener constante la temperatura de una estufa. 6. Un aspirador de aire con contador, y dos aparatos, de Gay-Lussac y de Dumas, para determinar la densidad de los vapores. 7. Un aparato de Berthelot para determinar el punto de ebullición de los líquidos y un calorímetro de Berthelot. 8. Representaciones gráficas de ondas, interferencias y de propagación de ondas en el agua. 9. Representaciones gráficas de las leyes de reflexión y refracción, de vibraciones luminosas y de composición o interferencias de dos rayos de luz. 10. Representaciones gráficas de interferencias de rayos de luz, refracción y dispersión, y doble refracción. 11. Representación gráfica de ejes ópticos y dibujos del prisma Nicol de espato de Islandia, y aparato de Biot y Arago para determinar el índice de refracción de los gases. 12. Sacarímetro de Soleil. 13. Sacarímetro de Laurent. 14. Espectroscopio de Kirchoff y Bunsen. 15. Plano del espectroscopio. 16. Microscopio de Carl Reichert, Viena. Estas láminas refuerzan el carácter del libro como obra pura de física.

Pero si he traído a escena este libro es por su relevancia para conocer el estado del análisis dimensional en la España del XIX. La importancia del tema 
le parecía tal a Garagarza que en el párrafo segundo de la Introducción de su libro afirma lo siguiente:

La reforma introducida por los progresos de los estudios de la electricidad en el valor numérico de las dimensiones físicas, ha dado en estos últimos tiempos la base más racional para la medición de estas magnitudes, y nos ha inclinado á dar principio por ella al estudio de las diversas partes de esta ciencia, necesitada en la actualidad de unificar el valor de los fenómenos físicos.

Y en efecto, en la segunda sección, Unidades, del Cap. I, p. 31, Garagarza desarrolla el tema de las Dimensiones de las cantidades físicas. Dice al respecto:

Todas las cantidades físicas pueden deducirse de las tres cantidades fundamentales, cuyos símbolos son $L, M, T .{ }^{9}$ La relación que liga una cantidad derivada con una ó varias de las cantidades fundamentales, se llama dimensión de la cantidad. Así, por ejemplo, una superficie es igual al producto de dos longitudes, un volumen es el producto de tres longitudes; las dimensiones respectivas de estas cantidades son $L^{2}$ y $L^{3}$.

Pues bien, el Cap. II, que carece de título, está dedicado íntegramente a la presentación de las magnitudes derivadas, recogidas en cinco grupos, cuyos nombres hemos dado más arriba. Por su relevancia física empiezo con el Segundo Grupo, el de Cantidades y unidades mecánicas. La primera magnitud derivada es obviamente la velocidad de la que dice (p. 33) que tiene por dimensión $\left[L T^{-1}\right]$. O sea, jutiliza la notación de Maxwell! ${ }^{10}$ Le sigue la velocidad angular, cuyas «dimensiones son $\left[T^{-1}\right]$ » y aceleración, cantidad de movimiento y unidades de fuerza: «las dimensiones de la unidad de fuerza con $\left[\frac{M L}{T^{2}}\right]$ ó $\left(M L T^{-2}\right) . »($ p. 35). Le siguen las dimensiones del trabajo, energía cinética, momento de un par (pp. 36-37), y de calor, que «es una cantidad física de la misma naturaleza que la energía, y tiene las mismas dimensiones: $M L^{2} T^{-2}$. Se manifiesta en los cuerpos por crecimiento de temperatura.» (p. 38). Termina este grupo con la presión, la cual «tiene por dimensiones ó se representa por las fórmulas: $\frac{F}{S}=\left[M L^{-1} T^{-2}\right]^{»}$

9 Longitud, Masa y Tiempo.

10 La notación de Maxwell, fórmula dimensional entre corchetes, es la usual. Una explicación la da Ricardo San Juan (1945, p. 3), donde afirma que los símbolos que designan las dimensiones se encierran en paréntesis cuadrados «precisamente para indicar que no se manejan números.» 
El tercer grupo está dedicado a las unidades magnéticas. En él Garagarza presenta, explica y da las dimensiones de: la unidad de polo magnético: $\left[M^{1 / 2} L^{3 / 2} T^{-1}\right]$, intensidad del campo magnético: $\left[M^{1 / 2} L^{-1 / 2} T^{-1}\right]$, momento magnético: $\left[M^{1 / 2} L^{5 / 2} T^{-1}\right]$, magnetismo específico: $\left[M^{-1 / 2} L^{5 / 2} T^{-1}\right]$. El cuarto grupo está dedicado a unidades y cantidades eléctricas. Entre ellas están la unidad electrostática de cantidad: $\left[M^{1 / 2} L^{3 / 2} T^{-1}\right]$ y la unidad electrostática de diferencia de potencial: $\left[M^{1 / 2} L^{1 / 2} T^{-1}\right]$.

Los demás capítulos de Instrumentos y aparatos de Física están dedicados a temas de física, teórica y aplicada, entre los que se incluyen el rechazo del calórico, la defensa de la teoría ondulatoria de la luz, el éter, las relaciones entre éter y luz, el electromagnetismo, y muchos más. Lo desconcertante es que Garagarza no haya hecho referencia al libro Unidades Físicas, de Muñoz del Castillo, colega suyo en la Universidad Central de Madrid, publicado dos años antes.

\section{EL LIBRO UNIDADES FÍSICAS, 1890, DE JOSÉ MUÑOZ DEL CASTILLO}

El granadino José Muñoz del Castillo (1850-1926), tras su paso por Logroño y la Universidad de Zaragoza, fue Catedrático de Química Mecánica desde 1887 en la Facultad de Ciencias de la Universidad Central, y Presidente de la Sección de Ciencias Físico-Químicas y de la Sociedad Española de Física y Química y Jefe del Instituto de Radiactividad de la Universidad Central, cuyo laboratorio fundó a finales de 1903 (Herrán 2008, p. 68). Entre sus estudios dedicados a la radiactividad destacan, entre muchos otros: Programas de estudios experimentales sobre las sustancias radioactivas y la radioactividad, 1904, Radioactividad y radiobiología, 1919, La radioactividad en físico-química y en hidrología médica, 1905. Fue además autor de un Tratado Elemental de Física, 1882, que sería complementado ocho años después con la monografía Unidades Físicas, 1890, que es el primer libro dedicado al análisis dimensional, publicado pues treinta y dos años antes del libro de Bridgman. A este texto Néstor Herrán (2008, p. 59) sólo le dedica la siguiente referencia: «Dicho libro... consistía en una introducción a los sistemas métricos internacionales (sistema métrico y cgs) y al cálculo dimensional.»

En el Capítulo I Unidades y Sistemas de Unidades, $\S \S 2 \mathrm{~A}$ y 2B, pp. 6-7 de su libro, José Muñoz del Castillo (1890) introduce ya el concepto de ecuación de las dimensiones (hoy más conocida como fórmula dimensional) de una magnitud física:

2-A El estudio de la naturaleza, y las aplicaciones de los conocimientos naturales traen consigo la necesidad de medir cantidades de variada condición externa; pero ante la unidad que preside á los fenómenos cósmicos, debe ser ideal lógico el establecimiento de un sistema coordinado de unidades, tal que su base, desarrollo y encadenamiento tengan por mecanismo la elección arbitraria de un cierto nú- 
mero (el menor posible) de unidades fundamentales; la derivación de las restantes unidades de modo que resulten ser simples relaciones entre las fundamentales; y la definición de cada unidad derivada mediante una expresión matemática. B.- La fórmula que define una unidad derivada en función de una ó varias de las fundamentales, se llama ecuación de las dimensiones de dicha unidad derivada.

Tomando como ejemplo la unidad derivada de velocidad, el primer ejemplo de ecuación de dimensiones en función de las unidades fundamentales de longitud y tiempo es: Unidad de velocidad= unidad de longitud / unidad de tiempo. Y tomando como unidad el metro cúbico, las dimensiones de éste serán (p. 7):

$($ Unidad de volumen $)=($ metro $) \times($ metro $) \times($ metro $)=(\text { metro })^{3}$.

Y en p. 8 Muñoz del Castillo reconoce la «ventaja práctica que proporcionan las ecuaciones de las dimensiones cuando precisa relacionar unidades correspondientes en distintos sistemas.»

La Sección 6 es muy esclarecedora:

6. - A. - La elección de las unidades fundamentales es la verdadera clave del mérito del sistema coordenado á que las mismas pueden dar lugar, y el punto objeto de preferente estudio.

B.- He aquí las condiciones experimentales más importantes á que han de satisfacer las unidades fundamentales.

$1^{\text {a }}$. Deben elegirse cantidades susceptibles de una comparación muy exacta con las de su misma especie.

$2^{\text {a }}$. Esta comparación debe poder ser efectuada en cualquier época. Por consiguiente, toda unidad podrá dar origen á patrones iguales, no susceptibles de alteración en el transcurso del tiempo.

$3^{\text {a }}$. La propia comparación debe ser posible en todos los parajes. Lo cual exige que los patrones no experimenten variación alguna de magnitud si se los traslada de un sitio á otro.

$4^{\mathrm{a}}$. Dicha comparación debe poder ser ejecutada con facilidad y directamente. En lo que va envuelta la existencia de medios prácticos de copiar ó reproducir los patrones.

C.- Además es conveniente que las unidades fundamentales permitan definir con sencillez, y representar por ecuaciones de dimensiones no complicadas, las unidades derivadas.

El Cap. II está dedicado al denominado sistema terrestre, cuyas unidades fundamentales son: unidad de longitud: el metro; unidad de fuerza: el kilogramo; unidad de tiempo: el segundo sexagesimal. Sus unidades derivadas de carácter geométrico son: Unidad de superficie y Unidad de volumen, cuyas correspondientes ecuaciones de dimensiones son las arriba indicadas. Por su 
parte las unidades derivadas de carácter mecánico son: Unidad de velocidad, de aceleración, de masa, de energía o fuerza viva y de potencia mecánica, cuyas dimensiones ofrece. (Por cierto, Muñoz del Castillo utiliza indistintamente 'ecuación de dimensiones' y 'dimensiones'.)

El capítulo IV lo dedica Muñoz del Castillo al sistema cegesimal, del que dice lo siguiente: «De los varios sistemas propuestos como absolutos ha prevalecido, por acuerdo del Congreso internacional de electricistas celebrado en París en 1881, el de la Asociación Británica, llamado del centímetro-gramosegundo, ó, por abreviatura, del C. G. S.»

$\mathrm{Y}$ añade que las unidades fundamentales del sistema C G. S. son tres: Una unidad de longitud: el centímetro, Una unidad de masa: el gramo-masa, Una unidad de tiempo: el segundo, representadas respectivamente por los símbolos $L, M, T$.

Pues bien, entre las páginas 24 y 62 desarrolla todas las unidades derivadas cegesimales, reunidas en los grupos siguientes:

- Unidades derivadas geométricas: centímetro cuadrado, de dimensiones $L^{2}$, y el centímetro cúbico, de dimensiones $L^{3}$.

- Unidades mecánicas: Unidad de velocidad, Unidad de aceleración, Unidad de fuerza, Unidad de trabajo y de energía, Unidad de potencia mecánica.

- Unidades magnéticas: Unidad de polo, Unidad de campo magnético, Unidad de flujo de fuerza.

- Unidades electro-magnéticas: Unidad de corriente o unidad de intensidad de corriente, Unidad de cantidad, Unidad de resistencia, Unidad de fuerza electromotriz y también Unidad de diferencia de potencial, Unidad de capacidad, Unidad de trabajo eléctrico, ó de energía eléctrica, Unidad de potencia eléctrica.

Cada una de estas unidades derivadas es perfectamente definida, deducida y su correspondiente ecuación de dimensiones convenientemente explicada. El grupo de unidades electromagnéticas se completa con dos Notas, la III, pp. 54-55, y la IV, p. 56. Según éstas, este grupo se divide en el subgrupo de Unidades electrostáticas, que se forma a partir de las nociones de cantidad de electricidad y de corriente de un fenómeno electrostático, y estaría compuesto por las unidades de cantidad de electricidad, que es «la cantidad de electricidad que, colocada a la distancia de un centímetro de otra igual y del mismo nombre, la rechaza con la fuerza de una dina»; su ecuación de dimensiones, deducida de la ley de Coulomb, es $M^{1 / 2} L^{3 / 2} T^{-1}$; unidad de corriente: corriente que en la unidad de tiempo transporta la unidad de cantidad de electricidad y cuyas dimensiones son $M^{1 / 2} L^{3 / 2} T^{-2}$; unidad de diferencia de potencial «que 
existe entre dos puntos cuando al pasar de uno á otro la unidad de cantidad de electricidad representa el trabajo de un erg.» y cuyas dimensiones son $M^{1 / 2} L^{1 / 2} T^{-1}$; unidad de resistencia, que es «la resistencia de un conductor por el cual tarda un segundo en pasar la unidad de cantidad de electricidad cuando se mantiene entre los extremos una diferencia de potencial igual a la unidad» y cuyas dimensiones son $T L^{-1}$; y unidad de capacidad de un conductor, que es el «cociente de dividir la cantidad de electricidad de que está cargado por el potencial que esta carga le produce; por consiguiente, capacidad unidad será la de un conductor en que la unidad de cantidad de electricidad eleve el potencial una unidad», y sus dimensión es $L$. Mientras que el subgrupo de Unidades electrodinámicas, se forma a partir de fenómenos electrodinámicos como atracciones y repulsiones de conductores. Las dimensiones de la Unidad de intensidad de corriente son $M^{1 / 2} L^{1 / 2} T^{-1}$.

Basten estas ecuaciones de dimensiones para ilustrar la importancia que Muñoz del Castillo concede al análisis dimensional, que completa con la Nota VII, p. 62, donde consigna nombres y dimensiones de algunas unidades cegesimales «poco o nada usuales»: densidad, momento de un par, radián, velocidad angular, aceleración angular, momento de inercia, cantidad de movimiento angular, atracción en el centro, potencia de un centro, curvatura de una línea, curvatura específica de una superficie, unidad electromagnética de fuerza eléctrica, potencial de una hoja magnética, fuerza magnética, potencial magnético, momento magnético, intensidad de imantación, constante de Verdet y coeficiente de inducción.

\section{CONCLUSIÓN}

En este artículo me he propuesto llenar una laguna en la historia del análisis dimensional. He rehabilitado las figuras pioneras de Muñoz del Castillo y de Fausto Garagarza, prácticamente desapercibidos hasta ahora para los historiadores de la física, y he reivindicado al primero de ellos como autor del primer tratado sobre análisis dimensional en la historia de esta disciplina.

\section{REFERENCIAS BIBLIOGRÁFICAS}

BRIDGMAN, P. 1922, Dimensional Analysis. New Haven: Yale University Press.

BUCKINGHAM, E. 1914 «On Physically Similar Systems: Illustrations of the use of dimensional equations». Physical Review 4: 345-376

BUCKINGHAM, E. 1915, «The Principle of Similitude», Nature 96: 396-397

FOURIER, J. B. 1822, Théorie analytique de la chaleur. Paris: Firmin Didot, Pére 
et Fils.

HERRÁN, N. 2008, Aguas, Semillas y Radiaciones. El Laboratorio de Radiactividad de la Universidad de Madrid, 1904-1929. Madrid: CSIC

GARAGARZA, F. 1892, Programa de la Asignatura de Instrumentos y Aparatos de Física de Aplicación á la Farmacia. Con los principios fundamentales para el conocimiento de la asignatura. Madrid: Librería de la Viuda de Hernando y $\mathrm{C}^{\mathrm{a}}$.

GONZÁLEZ DE POSADA, F. 2002, «Génesis histórica, enunciado y evolución del análisis dimensional de Julio Palacios». Llull 25: 399-423

GONZÁLEZ REDONDO, F. 2000, Historia del Análisis Dimensional. Tesis Doctoral. Universidad Politécnica de Madrid. Escuela Técnica Superior de Arquitectura.

MARTINS, R. 2008, «A busca da ciência a priori no final do século XVIII e a origem da análise dimensional». En: Martins, R. A. et al. (eds.), Filosofía e História da Ciência no Cone Sul. $3^{\circ}$ Encontro. Campinas: AFHIC, 391-402

MAXWELL, J. C. 1862-1873, Scientific Letters and Papers. Edited by P. H. Harman. Volume II. Cambridge: University Press, 1995

MAXWELL, J. C. 1874-1879, Scientific Letters and Papers. Edited by P. H. Harman. Volume III. Cambridge: University Press, 2002

MAXWELL, J. C. 1871, Theory of Heat. With corrections and additions (1891) by Lord Rayleigh. New impression, London: Longmans, Green, and Co. 1908

MAXWELL, J. C. 1873, A Treatise on Magnetism and Electricity. Oxford: Clarendon Press

MAXWELL, J. C. 1877, «On Dimensions». En J. C. Maxwell, Scientific Letters and Papers, Vol. III, 517-520.

MONOD-HERZEN, G. 1976, L'analyse dimensionelle et l'épistémologie. Paris: Maloine Éditeur.

MUÑOZ DEL CASTILLO, J. 1890, Unidades Físicas. Madrid: Celestino Apaolaza

PALACIOS, J. 1964, Análisis Dimensional. Segunda edición corregida y aumentada. Madrid: Espasa-Calpe. Primera edición 1955

PORTER, A. 1933, The method of dimensions. London: Methuen

RAYLEIGH, L. 1915, «The Principle of Similitude», Nature No. 2368, vol. 95, March 18: $66-68$

RIVADUlLA, A. 2015, Meta, Método y Mito en Ciencia. Madrid: Ed. Trotta

SÁNCHEZ RON, J. M. 1992, «Las ciencias físico-matemáticas en la España del siglo XIX». En José M. López Piñero (ed.), La ciencia en la España del Siglo XIX. Madrid: Marcial Pons

SÁNCHEZ RON, J. M. (2003): «La Física en España (I): el Siglo XIX». Revista Española de Física, Enero-Febrero de 2003: 5-9

SAN JUAN, R. 1945, Teoría de las Magnitudes Físicas y sus Fundamentos Algebraicos. 
Revista de la Real Academia de Ciencias de Madrid, 39. Madrid: C. Bermejo.

Andrés Rivadulla es catedrático de Lógica y Filosofía de la Ciencia. Profesor Honorífico de la Facultad de Filosofía de la Universidad Complutense de Madrid.

Líneas de Investigación:

Metodología de la Ciencia y Epistemología, Historia y Filosofía de la Física, Historia y Filosofía de la Probabilidad y de la Estadística Teórica.

Publicaciones recientes:

(2015): Meta, Método y Mito en Ciencia. Madrid: Editorial Trotta.

(2016): «Models, Representation and Incompatibility. A Contribution to the Epistemological Debate on the Philosophy of Physics». J. Redmond et al. (eds), Epistemology, Knowledge and the Impact of Interaction. Springer International Publishing Switzerland, pp. 521-532.

Correo electrónico: arivadulla@filos.ucm.es 